\title{
Colour Adjustment and Specular Removal for Non-Uniform Shape from Shading
}

\author{
Xiaozheng Zhang $^{\dagger *}$, Yongsheng Gao ${ }^{\dagger *}$, Terry Caelli ${ }^{\dagger}$ \\ ${ }^{\dagger}$ Biosecurity Group, Queensland Research Lab, National ICT Australia \\ \{paul.zhang, yongsheng.gao, terry.caelli\}@nicta.com.au, \\ $\$$ Computer Vision and Image Processing Lab, Griffith University, Australia \\ $\{x . z h a n g$, yongsheng.gao\}@griffith.edu.au
}

\begin{abstract}
Surface colour changes and specular reflections are two major problems in $3 D$ modelling using shape-from-shading (SFS). This paper proposes to pre-process the input image for a typical SFS algorithm, so that the resultant image has no colour changes and specular reflection. First, a chromaticity-based specular reflection removal algorithm is applied to achieve a pure diffuse (Lambertian) reflected image. Then, a novel chromaticity-based colour adjustment approach is proposed to generate an image without surface colour changes. The standard SFS algorithms can then be applied successfully onto the processed images to produce plausible $3 D$ models. In experiments, the proposed approach was tested on standard SFS datasets with complex surface colours. The experimental results show it's promising to facilitate SFS algorithms to handle SFS problems with more complex surface properties and illumination conditions.
\end{abstract}

\section{Introduction}

Shape-From-Shading (SFS) recovers 3D structure information from a single 2D image, which has attracted a large number of research efforts dating back to 1960s [3]. For comprehensive reviews, please refer to the two excellent survey papers on this topic $[2,8]$. Despite the continuous efforts, many theoretical and technical problems still remain open due to the ill-posed nature of shape-from-shading. Among them, specular reflection and surface property changes are two of the prominent problems for typical shape-from-shading, as a typical shape-from-shading algorithm assumes Lambertian surface reflectance model with a uniform albedo along the surface. These two important constraints help to keep the shape-from-shading problem linear and tractable.

Recently, a few attempts have been made towards lifting one of these assumptions. Ragheb and Hancock [4] proposed to solve a specular shape-from-shading problem within a probabilistic framework with introduction of prior shape constraints and sacrifice of computational stability as the problem becomes non-linear. Shape-from-shading has also been applied to model surfaces with complex (non-uniform) surface properties, such as faces $[1,6]$.

In this paper, we propose to handle both of the two problems in shape-from-shading in an alternative way. Instead of covering specular reflection and surface non-uniformity in the shape-from-shading calculation, this paper proposes to convert the input 2D image with specularity and surface changes to an image of a diffuse-only uniform surface. After this pre-processing, typical shape-from-shading algorithms can then perform well on the adjusted image. The proposed approach uses chromaticity of colour input images as the cue to classify specular reflections and colour (surface property) discontinuity. The entire image is segmented based on chromaticity into different regions with same surface colours. In each region, the specular reflection can be calculated in a least square sense. The colour adjustment parameters are then calculated inter-regionally in a least square sense. The resultant image is then specular-free and with a uniform surface colour, whose shape is identical to the shape of the original input image. With the proposed colour adjustment and specular removal, typical shape-from-shading can be applied without any alteration to handle images with specular reflection and colour changes.

The rest of the paper is organised as follows. Section 2 shows the effects of the presence of specular reflection and colour changes in shape-from-shading modelling. The proposed approach is elaborated in Section 3. The experiments on standard shape-from-shading testing datasets are presented in Section 4. The conclusions are given in Section 5.

\section{Colour changes and specular reflection in shape-from-shading}

In this paper, we select Tsai and Shah's method [8] as the testing shape-from-shading algorithm, which is a linear 
approach based on Lambertian and uniformity assumptions. To illustrate the effects of modelling surfaces with colour changes and specular reflection, the standard testing object, vase, is used for rendering input images and shape-from-shading modelling. Figure 1 shows the ground truth of the vase shape.

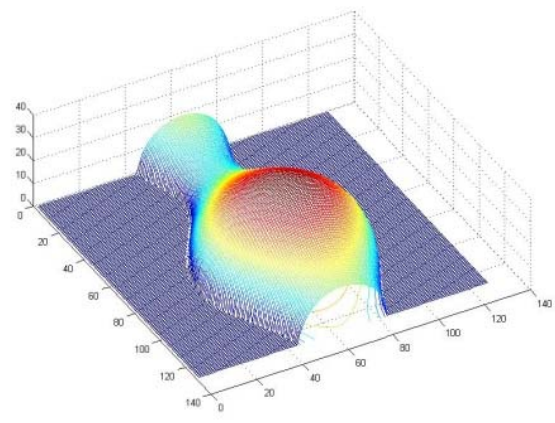

Figure 1: The ground truth shape of the vase.

Using the standard diffusely rendered vase image with a frontal light, the shape can be well modelled using Tsai and Shah's method as shown in Figure 2. The input image is directly from [8]. To show the effect of specular reflection, a new image is rendered with a Phong surface model on the vase with the same illumination conditions. The new input image as shown in Figure 3(a) is first converted to grey-scale image (Figure $3 b$ ), then modelled using Tsai and Shah's method. The modelling result is shown in Figure 4, which contains big deviations from the ground-truth shape on the positions of the specular reflections. This test shows that the specular reflection affects the faithfulness of shape-from-shading modelling adversely.

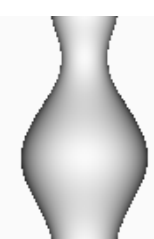

(a)

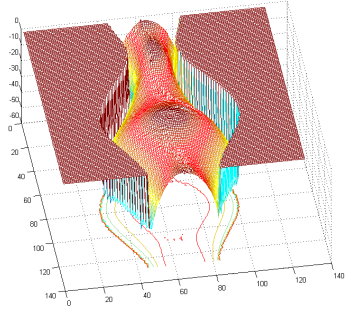

(b)
Figure 2: The shape-from-shading modelling of vase without specular reflection and colour changes. (a) The standard input image of the vase, and (b) the modelling result.

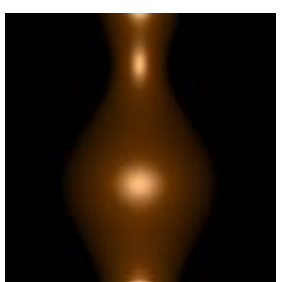

(a)

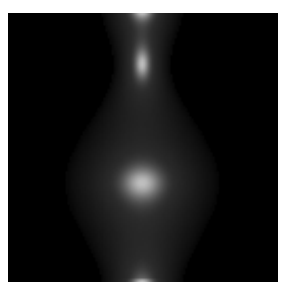

(b)
Figure 3: The rendered vase image with specular reflection. The surface is approximated using Phong model containing both diffuse specular reflectance coefficients. (a) colour image, and (b) converted grey-scale image.

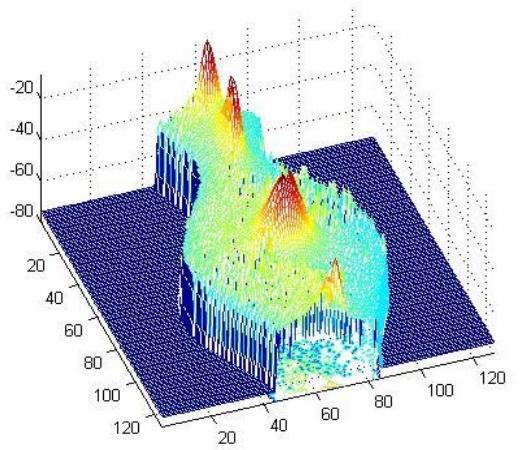

Figure 4: The modelling result from Figure 3(b). Big shape deviations occur on the positions of specular reflections.

The surface colour changes are another problem in shape-from-shading. To illustrate this effect, two images are rendered. One is a vase with uniform surface colour, while the other is the same vase with two surface colours in a chess board style. These two images are shown in Figure 5(a) and 5(b), respectively. For the vase with colour changes, the two colours are red $(255,0,0)$ and green $(0$, $255,0)$. Similar to the previous modelling, both images are first converted to grey-scale images. Applying shape-from-shading algorithm, the modelling results are shown in Figure 6(a) and 5(b), respectively. Comparing the two modelling results, the vase with a uniform surface colour has no shape discontinuity as existing in the vase with colour changes. In shape-from-shading, due to the uniform colour assumption, the surface colour changes are transferred or interpreted as shape changes.
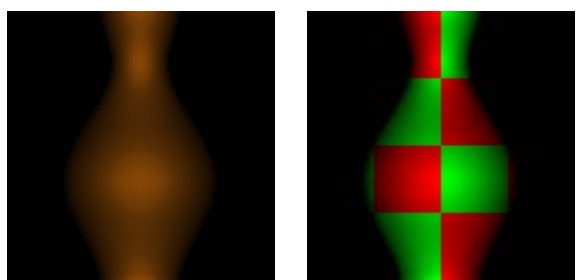

Figure 5: The rendered vase image without or with colour changes. (a) a uniformly coloured vase image, and (b) a vase image with a colour changes.

These two tests have shown that the specular reflection and colour changes are two prominent problems in shape-from-shading modelling. These two issues will cause the modelling results very different from the ground truth. As most of the typical shape-from-shading algorithms make the assumptions of Lambertian surfaces and uniform surface colours, these effects shown in Figure 4 and Figure 6 are not unique for a specific shape-from-shading 
algorithm, but a quite common problem for a group of shape-from-shading algorithms.

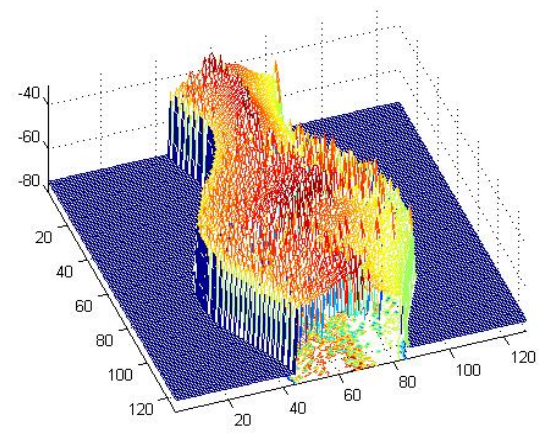

(a)

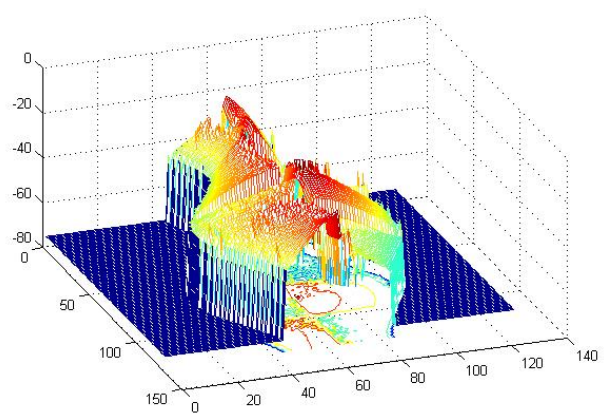

(b)

Figure 6: The modelling result from Figure 5(a) and 5(b), respectively. (a) the modelling result of a uniform colour vase, and (b) the modelling result of a vase with surface colour changes. Shape discontinuities occur on the edges of surface colour discontinuities.

\section{Specular removal and colour adjustment}

To overcome the effects of specular reflection and colour changes, we propose to pre-process the input images with specular removal and colour adjustment. First, a chromaticity-based specular removal is applied on the colour input image with specular reflections.

\subsection{Specular removal}

Recently, a few methods have been proposed to separate diffuse and specular reflection based on chromaticity analysis, such as $[5,7]$. These methods do not require the knowledge of lighting directions and object geometries. They only use chromaticity, the ratios of different colour channels (i.e., red, green, blue in RGB colour system), as the cue to classify similar colour regions.

To remove specular reflection, the diffuse reflections are labelled for each pixel. First, a specular-free image is generated as follows and used as a reference image for classifying diffuse and specular reflections.

$$
V_{s f, i}(p)=V_{i}(p)-V_{\min }(p)+\bar{V}_{\text {min }}
$$

where $V_{i}(p)$ is one colour channel of point $p, i=1,2,3$, representing red, green and blue, respectively, $V_{\min }(p)=$ $\min \left(V_{i}(p)\right)$ is the colour channel with minimum intensity, $\bar{V}_{\text {min }}$ is the average $V_{\min }(p)$ for the entire image.

The diffuse reflection region is then classified using the reference specular-free image $V_{s f}$ if $V_{i}(p)-V_{s f, i}(p)<$ th1. Otherwise, the pixel is classifies as pixels containing both diffuse and specular reflections. The threshold th 1 is chosen as $\bar{V}_{\min }$. For the pixels with only diffuse reflections, there is no need to remove specular reflection. These pixels are used as reference pixels for the other pixels with both diffuse and specular reflections. To classify similar colour regions, the chromaticity of the specular-free image is used as follows.

For a point $p$ in the diffuse region, classify all pixels $\{q\}$ in the diffuse and specular regions which satisfying

$$
\sum\left|\mathrm{c}_{\mathrm{sf}, \mathrm{i}}(\mathrm{q})-\mathrm{c}_{\mathrm{sf}, \mathrm{i}}(\mathrm{p})\right| \leq \mathrm{th} 2,
$$

where $c_{s f, i}(p)=V_{s f, i}(p) / \sum V_{s f, i}(p)$ is the chromaticity of the specular-free image $V_{s f}$. For each pixel $q$, the diffuse and specular components can be calculated using the diffuse component of $p$ as

$$
\boldsymbol{V}(q)=\alpha(q) \boldsymbol{V}(p)+\beta(q)\left[\begin{array}{l}
1 \\
1 \\
1
\end{array}\right]
$$

where $\alpha$ and $\beta$ are diffuse and specular coefficients, respectively. The diffuse component of pixel $q$ is then calculated as $\boldsymbol{V}_{d}(q)=\alpha(q) \boldsymbol{V}(p)$.

Figure 7-9 show the process of the specular removal and diffuse/specular separation of the vase image with specular reflection. Figure 7 is the input vase image with specular reflection. Figure 8(a) shows the generated specular-free reference image. Figure 8(b) shows the diffuse only pixels, which do not have specular reflection. Figure 9 shows the resultant images of diffuse and specular reflection separation. Figure $9(\mathrm{a})$ is the diffuse component after separation and Figure 9(b) is the specular component of the original image (Figure 7). Specular removal is equivalent to generating Figure 9(a).

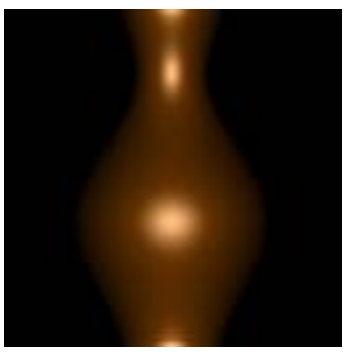

Figure 7. The input vase image with specular reflection. 


\subsection{Colour adjustment}

To alleviate the effects caused by colour changes, we propose a novel chromaticity-based colour adjustment on the input colour image for shape-from-shading. The proposed colour adjustment is based on the following assumptions. First, the input colour image only contains diffuse reflection obeying Lambertian surface model. If the input colour image contains specular reflection, we can perform the specular removal algorithm introduced in Section 3.1. Second, the same chromaticity represents the same surface colour. This is similar assumption to the specular removal as in specular removal, the chromaticity is used as a cue to classify colour regions. Thirdly, there is no big achromatic (grey-scale) area in the image.
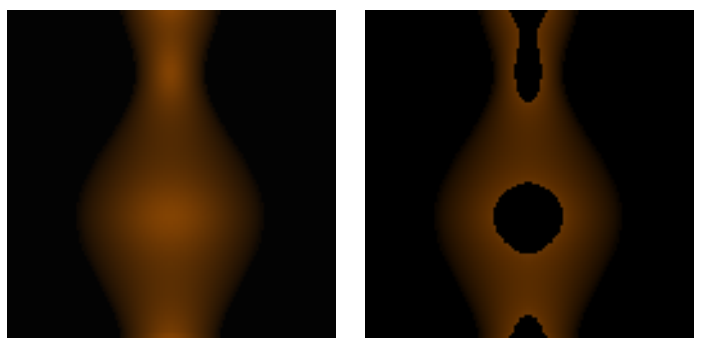

Figure 8 . The intermediate images. (a) the specular-free reference image, and (b) the image showing diffuse only pixels.
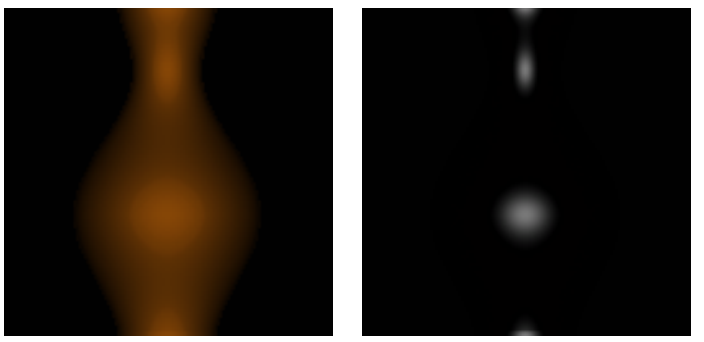

Figure 9. The separation results. (a) the diffuse component of Figure 7, and (b) the specular component of Figure 7.

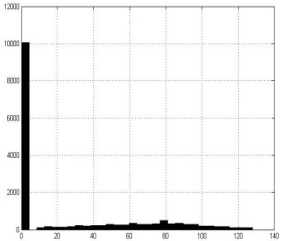

(a)

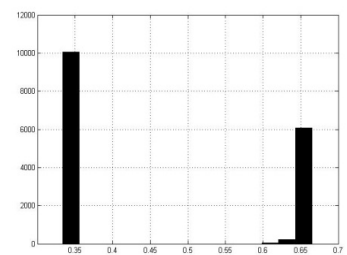

(b)
Figure 10. The histogram (Figure 5a) of (a) the red intensity, and (b) the red chromaticity. The red intensity changes as different pixels have different surface orientations. But the chromaticity (the right bar) remains the same independent to the different shape conditions.

The proposed colour adjustment is based on an observation that chromaticity is shape independent. Assuming a white illumination and a balanced camera reception, the changes of illumination intensity and surface orientations only cause all of the 3 colour channels to change proportionally. As chromaticity is the ratio of the colour channels, it does not change under different illumination intensity and shape changes. Figure 10 and Figure 11 show the difference between pixel intensity and chromaticity, as pixel intensity changes due to illumination and shape changes. Figure 10 shows the histograms of the red intensity and red chromaticity of Figure 5(a), while Figure 11 shows $3 \mathrm{D}$ histograms of the red-green intensity and the red-green chromaticity. The high bars in histograms represent the black pixels.

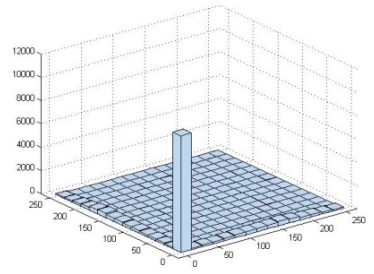

(a)

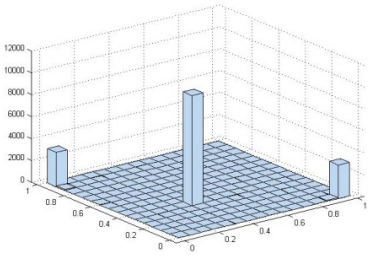

(b)

Figure 11. The 3D histogram (Figure 5b) of (a) the red-green intensity, and (b) the red-green chromaticity.

Based on the observation that the chromaticity is shape independent, we propose to use chromaticity as colour region classification cue so that it won't be affected by shape. For a point $p$, a region $\{q\}$ can be classified as all pixels which satisfy

$$
\sum\left|\mathrm{c}_{\mathrm{i}}(\mathrm{q})-\mathrm{c}_{\mathrm{i}}(\mathrm{p})\right| \leq \text { th, }
$$

where $t h$ is a colour separation threshold and in this paper, we use $t h=0.1$. For the image of Figure 5(b), different regions can be classified and labelled as shown in Figure 12.

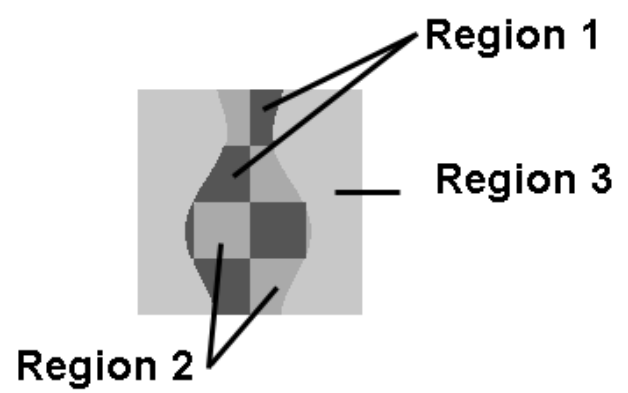

Figure 12. The classification of different colour regions using chromaticity cue. 
To adjust colour, a scaling parameter $a_{j}$ is assigned to colour region $j$. The colour in region $j$ is adjusted as

$$
V_{i}^{\prime}(p)=\left(1+a_{j}\right) V_{i}(p)
$$

where $V_{i}^{\prime}$ is the adjusted colour intensity. To enforce the surface colour to be uniform in grey-scale image, the brightness on the edge of two different regions should be continuous. We first convert the colour image into grey-scale image using the empirical conversion equation

$$
G(p)=0.30 V_{1}(p)+0.59 V_{2}(p)+0.11 V_{3}(p) .
$$

The neighbouring pixels $p$ and $q$ should satisfy

$$
[1+a(R(p))] G(p)=[1+a(R(q))] G(q),
$$

if $p$ and $q$ belong to two different regions. $R(p)$ represents the region label of pixel $p$. Find all pixel pairs $(p, q)$ which are neighbours and belong to different regions. There will be $n$ equations in the style of (7), where $n$ is the number of such pixel pairs. For different regions correspond to different scaling parameters, the $n$ equations can be represented in a matrix form as

$$
M \cdot\left(a_{1}, a_{2}, \ldots, a_{r}\right)^{T}=b .
$$

For the $k$ th pixel pair $(p, q)$,

$$
\left\{\begin{array}{l}
M(k, R(p))=G(p) \\
M(k, R(q))=-G(q) . \\
b(k)=G(q)-G(p)
\end{array}\right.
$$

Equation (8) is solved in a least square sense and the scaling parameters $a_{j}$ can be obtained. Then the colour is adjusted using Equation (5) and the grey-scale image is generated using Equation (6). Figure 13 shows the colour adjusted image of Figure 5(b). It shows that in grey-scale image, the input image becomes uniform and is hence suitable for shape-from-shading.

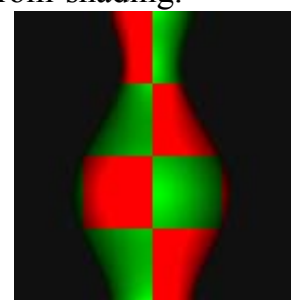

(a)

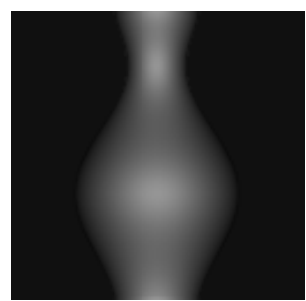

(b)
Figure 13. The results of colour adjustment of Figure 5(b). (a) the colour image, and (b) the grey-scale image after converting using Equation(6).

\section{Experiments}

In experiments, we selected the standard testing object, the vase, for shape-from-shading and rendered it with specular reflection and colour changes. The rendering without specular reflection and without colour changes were used as ground truth for shape-from-shading. The Tsai and Shah's shape-from-shading method was used as the testing algorithm for 3D modelling. In the first session, we compared the effectiveness of specular removal for shape-from-shading. Figure 14(a) is rendered with specular reflection under Phong surface model, while Figure 14(b) is the rendered image with diffuse reflection only. Figure 14(c) shows the result of specular removal from Figure 14(a). Figure 15 shows the modelling results of shape-from-shading using images of Figure 14. It shows that the specular removal helps shape-from-shading algorithm to handle input images with specular reflections.
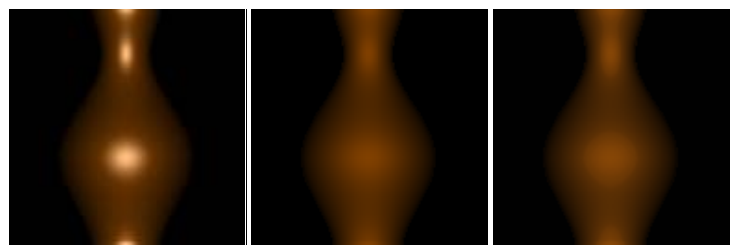

Figure 14. The input images before and after specular removal. (a) the image with specular reflection, (b) the diffuse image, and (c) the image after specular removal from (a).

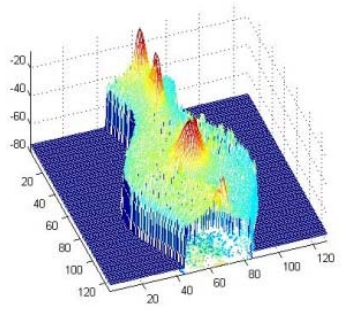

(a)

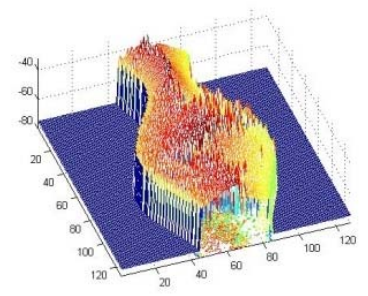

(b)

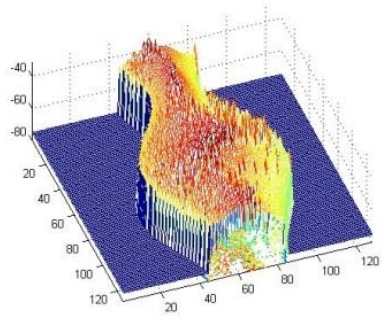

(c)

Figure 15. The shape-from-shading modelling results of (a) Figure 14(a), (b) Figure 14(b), and (c) Figure 14(c).

In the second session, we investigated the effectiveness of the proposed colour adjustment using the vase object. The vase object was rendered in a chess board style with two surfaces colours. Figure 15 shows the input image and modelling result. It can be seen that on the colour discontinuity, there exist shape discontinuities in the $3 \mathrm{D}$ 
model. Figure 16 shows the colour adjusted image and modelling result, which is much smoother than the original image. Further, we have randomly pigmented the chess board on the vase as shown in Figure 17 with modelling result. The colour adjusted counterpart is shown in Figure 18. As shown in Figure 18(b), the colour adjustment brought the different brightness caused by different surface colours to a uniform surface colour in the grey-scale image, which enables the shape-from-shading to function normally.

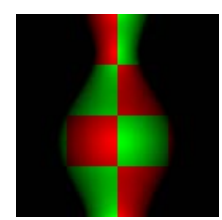

(a)

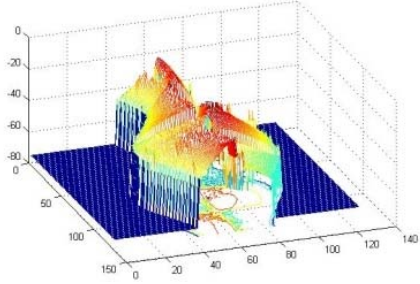

(b)
Figure 16. The original input image (a) and the SFS modelling result (b).

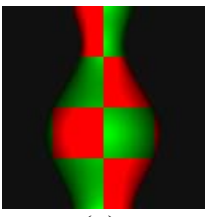

(a)

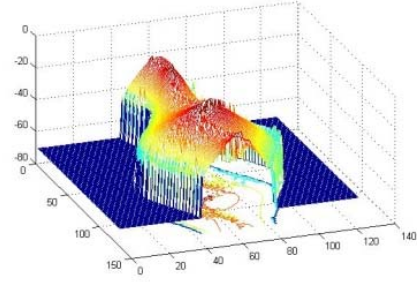

(b)
Figure 17. The colour adjusted image (a) and the SFS modelling result (b).
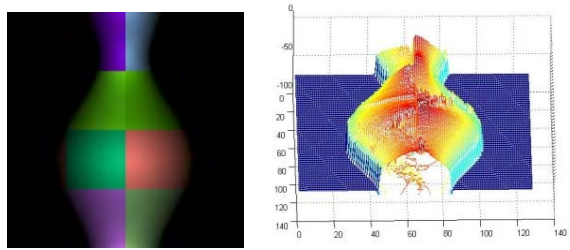

Figure 17. The original input image and the SFS modelling result.
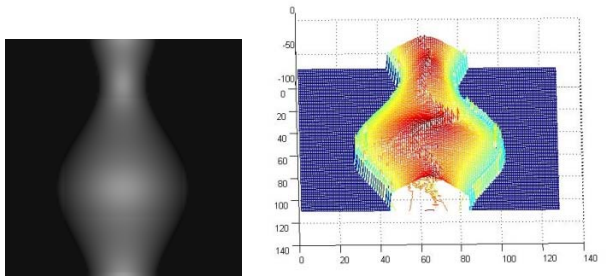

Figure 18 The converted grey-scale image, and the SFS modelling result.

\section{Conclusions}

In this paper, we investigated the effects of specular reflection and colour changes in the $3 \mathrm{D}$ modelling using shape-from-shading. Both caused negative influence to the qualities of the modelling. Different from including these two effects in the modelling process, this paper proposed to pre-process the input images with two steps, 1) specular removal and 2) colour adjustment. Both processes are based on chromaticity as it is independent to illumination changes and shape related surface orientation changes. Therefore, both processes do not require any knowledge of illumination and object geometry.

In both processes, the colour regions were classified using chromaticity of pixels. The specular components and the colour adjustment scaling parameters were calculated in least square senses in the colour region. Using the resultant images after specular removal and colour adjustment, shape-from-shading algorithms performed normally on the images with specular reflections and surface colour changes. In experiments, the standard vase object was rendered with specular and colour changes. The proposed approach was successfully applied on the input images and both specular reflection and colour changes were removed. The resultant images are diffuse only images with uniform surface properties, which are suitable for shape-from-shading to operate. The experimental results showed that the proposed colour adjustment and specular removal improved the shape-from-shading modelling results significantly. It is a promising method to bring the typical shape-from-shading algorithms to more general use scenarios.

\section{References}

[1] R. Dovgard and R. Basri, "Statistical symmetric shape from shading for 3D structure recovery of faces," in 8th European Conference on Computer Vision, vol. 2, 2004, pp. 99-113.

[2] J.-D. Durou, M. Falcone, and M. Sagona, "Numerical methods for shape-from-shading: A new survey with benchmarks," Comput. Vis. Image Understand., vol. 109, no. 1, pp. 22-43, 2008.

[3] B. K. P. Horn and M. J. Brooks, Shape from shading. MIT Press, 1989.

[4] H. Ragheb and E. R. Hancock, "A probabilistic framework for specular shape-from-shading," Pattern Recognition and Data Mining, Pt 1, Proceedings, vol. 36 no. 2, pp. 407-427, 2003.

[5] H.-L. Shen, H.-G. Zhang, S.-J. Shao, and J. H. Xin, "Chromaticity-based separation of reflection components in a single image," Pattern Rec., vol. 41, no. 8, pp. 2461-2469, 2008.

[6] W. A. P. Smith and E. R. Hancock, "Estimating facial reflectance properties using shape-from-shading," Int. J. Comput. Vision, vol. 86, pp. 152-170, 2010.

[7] R. T. Tan and K. Ikeuchi, "Separating reflection components of textured surfaces using a single image," IEEE Trans. Pattern Anal. Mach. Intell., vol. 27, no. 2, pp. 178-193, 2005.

[8] R. Zhang, P.-S. Tsai, J. E. Cryer, and M. Shah, "Shape-from-shading: a survey," IEEE Trans. Pattern Anal. Mach. Intell., vol. 21, no. 8, pp. 690-706, 1999. 